REVISTA CHILENA DE DERECHO DEL TRABAJO Y DE LA SEGURIDAD SOCIALC, VOL 6, N 12, 2015, pp. 178-185

Víctor Ricardo Juri Sabag / Alcances sobre las multas aplicadas por la Dirección del Trabajo y su reclamación. Comentario a Sentencia del Primer Juzgado del Trabajo de Santiago RIT I-15-2015

\title{
ALCANCES SOBRE LAS MULTAS APLICADAS POR LA DIRECCIÓN DEL TRABAJO Y SU RECLAMACIÓN. COMENTARIO A SENTENCIA DEL PRIMER JUZGADO DEL TRABAJO DE SANTIAGO RIT I-15-2015
}

\author{
SCOPES ON THE FINES APPLIED BY THE DIRECTION OF THE \\ WORK AND HIS CLAIM. COMMENT TO JUDGMENT OF THE FIRST \\ LABOR COURT OF SANTIAGO RIT I-15-2015
}

\author{
VÍCTOR RICARDO JURI SABAG*
}

\begin{abstract}
RESUMEN: En virtud de su función fiscalizadora del cumplimiento de las normas laborales y previsionales, la Dirección del Trabajo aplica multas a las empresas en caso de incumplimiento de dichas normas, tema que no esta exento de dificultades, contradicciones, ilegalidades y arbitrariedades, que se comprueban en los procesos y sentencias de reclamo de multas ante los tribunales laborales.
\end{abstract}

Palabras claves: Fundamentos de resoluciones; principio de "non bis idem"; reclamación de multas.

ABSTRACT: By virtue of his scrutinising role of the fulfillment of the labor procedure and social security, the Direction of the Work, it applies fines to the companies in case of breach of the above mentioned procedure, topic that not I exempt this one from difficulties, contradictions, illegalities and arbitrariness, which are verified in the processes and judgments of claim of fines before the labor courts.

Key words: Foundations of resolutions, "non bis idem" notions, claim of fines.

\section{INTRODUCCIÓN}

En este trabajo se abordaran los siguientes temas:

Los fundamentos de las resoluciones que aplican las multas y su eventual revocación por carecer de ellos.

La aplicación de más de una multa por el mismo tipo de infracción, lo cual se relaciona con el principio "non bis in ídem"

El plazo para reclamar administrativamente las multas, que establece el artículo 512 del Código del Trabajo, ¿es de días corridos o hábiles?

\footnotetext{
* Abogado. Licenciado en Ciencias Jurídicas y Sociales de la Universidad de Chile. Magíster en Derecho Tributario (LL.M.) de la Universidad de Chile. Profesor de Derecho del Trabajo de la Universidad de Chile. Contacto: rjuri@live.cl
} 


\section{LOS FUNDAMENTOS DE LAS RESOLUCIONES QUE APLICAN LAS MULTAS Y SU EVENTUAL REVOCACIÓN POR CARECER DE ELLOS}

En primer lugar, hago presente que las resoluciones que aplican las multas, como toda fiscalización y resolución administrativa debe ser fundada, de acuerdo a los artículos 16 y 41 de la ley 19.880.

En este tema, es interesante analizar la sentencia de la causa RIT: I-15-2015, del Primer Juzgado del Trabajo de Santiago, que resolvió un reclamo judicial de una multa aplicada por no pagar la remuneración mensual. El fiscalizador no consideró un pacto de suspensión de las obligaciones de prestar servicios y de pagar la remuneración íntegra ya que, se acordó que ese mes en que las manipuladoras de alimentos de un establecimiento educacional municipal, no tenía labor que realizar con motivo de las vacaciones escolares, el empleador les pagaría un bono de monto inferior a la remuneración mensual.

El empleador reclamó judicialmente la multa basado en un error de hecho del fiscalizador y pidió se dejara sin efecto la multa.

El fallo, acogió el reclamo y dejó sin efecto la multa, basado en:

\subsection{EL PRINCIPIO DE PROTECCIÓN AL DEBIDO PROCESO.}

Este principio se materializa - entre otras situaciones - en el derecho de toda persona a exigir el fiel cumplimiento de que toda resolución como del proceso administrativo que le dio origen, tenga los fundamentos mínimos en los cuales descansa la decisión del órgano sancionador. Así, este derecho, explica el sentenciador, lleva implícito la prerrogativa a ser oído y a que en su beneficio, se establezca la debida bilateralidad, unida a la transparencia y publicidad de las actuaciones administrativas. A lo anterior, se suma la obligatoriedad de fundamentación de las resoluciones por los entes administrativos. Así, en base de lo anterior, es que se puede ejercer adecuadamente el control sobre su actuar. Lo anterior, no sólo guarda relación con la legislación laboral, sino que además, es propio de los actos administrativos, conforme lo dispone la Ley $\mathrm{N}^{\circ}$ 19.880, en sus artículos 16 y 41. De esta suerte, se debe concluir que la autoridad administrativa debe encuadrar su actuar a un proceso con apego estricto a la conducta típica penada, esto es, previo a aplicar el castigo, debe realizar la descripción de la conducta infractora de la legislación laboral, permitiendo al sancionado articular su defensa en base a argumentaciones jurídicas y fácticas ciertas, exigencia que en definitiva permite distinguir el ejercicio propio de las facultades que la Constitución y las leyes entregan al ente estatal de un acto de mero arbitrio. 
De la prueba aportada al proceso, especialmente de la multa cursada -que es la que tipifica la sanción administrativa y por tanto, constata el hecho ilegítimo-, de modo alguno se puede desprender cuáles fueron los lineamientos de razonabilidad tenidos en consideración por el fiscalizador al determinar una infracción a la legislación laboral. A este respecto, se debe recordar que la infracción constatada textualmente señala "no pagar la remuneración de las manipuladoras de alimentos" (sic) sólo indicando la normativa legal supuestamente infringida en el recuadro siguiente. A lo anterior, se debe sumar que de los documentos tenidos a la vista por el fiscalizador, unidos a los requeridos $-\mathrm{y}$ exigidos por el reclamante, en el proceso administrativo-, tuvieron los fundamentos mínimos para desprender la hipótesis infractora, de suerte tal que su conclusión debió de contener aquellos mínimos lineamientos de razonabilidad en los cuales se sustentaba la imputación, cosa que como se ha señalado, no ocurrió. En efecto, en ninguna parte de la multa ni del informe de fiscalización, se desprende la forma en que la documentación exhibida, en los hechos, constituía una infracción, sólo basándose en consideraciones normativas de orden legal y administrativas, para suponer que se daban por infringidas las reglas sobre remuneración que en lo pertinente, dicen relación con el pago por los meses de enero y febrero.

De esta forma, independientemente del fondo del asunto, lo cierto es que la decisión del órgano administrativo fiscalizador no indicó de qué forma entendió que las normas laborales se han visto infringidas, pues, como se ha señalado, no basta con indicar un precepto normativo, sino que es menester además la adecuada fundamentación del mismo. Es preciso señalar que tal como lo señala Norberto Bobbio, las normas jurídicas no existen nunca solas, sino siempre en un contexto de normas que tienen entre sí relaciones particulares y este contexto de normas se acostumbra denominar ordenamiento. Se ha definido al ordenamiento jurídico como el conjunto unitario y coherente de normas que rigen en un cierto momento dentro de un ámbito espacial determinado y se trata, entonces, de una realidad normativa y, como "ordenamiento" que es, las normas jurídicas que lo componen deben estar vinculadas unas con otra coordinada o subordinadamente. Los principios generales del derecho o “espíritu general de la legislación” en los términos del artículo 24 del Código Civil, forman parte del ordenamiento jurídico, viven en su interior e informan sus normas e instituciones. De esta forma es preciso comprender que un órgano, específico y capacitado, que se encuentra dotado de la facultad sancionadora, depende en su actuar de los lineamientos mínimos de un Estado Republicano. Así, desde el instante en que se verifica un hecho se debe describir la forma en que tal o cual conducta infringe aquel compendio normativo, amén de que la misma conlleve la debida tipificación y explicitación de la forma en que su actuar vulnera el marco normativo. Sostener lo contrario - esto es, la no existencia de fundamentación al momento de aplicar una sanción administrativa-, no solo importaría desapegarse del mandato Constitucional de un justo y racional proceso, sino que dejaría a los ciudadanos en la indefensión, pues impediría el control de los órganos judiciales en cuanto a decisiones adoptadas. 
De esta suerte y en ésta línea argumentativa - sin adentrarse al fondo - se puede concluir que la empresa nunca tuvo cabal conocimiento de los reproches al incumplimiento de la ley laboral, pues no se explicitaron la forma en que sus conductas fueron y han sido objeto de ilícito laboral pues, la sola determinación de un artículo no implica fundamentación.

A mayor abundamiento, continua el fallo, debe entenderse que lo que se hace en una resolución de multa, lo que hace el ente sancionador, es interpretar las normas a aplicar, dándoles el sentido y alcance que considera debe darse, a fin de armonizar las diversas normas en juego, con la libertad de las partes para contratar, sobre todo en el caso de funciones en que tanto el empleador como el trabajador acordaron una forma de remuneración claramente determinada.

Indicó el sentenciador que, con la restante prueba instrumental, en especial las copias liquidaciones de remuneraciones y el anexo incorporado, se desprende que de modo alguno la remuneración acordada por los contratantes fue dejada sin efecto, pues el permiso, en beneficio de aquellos trabajadores, les permitía disponer incluso de un monto superior al normalmente percibido, pues les dejaba la posibilidad de efectuar otras labores, lo que unido a la continuidad de sus funciones - pues disponían de contrato indefinido, protegía íntegramente aquellos derechos que supuestamente fueron vulnerados, como ha sostenido el ente fiscalizador. De esta suerte, este sentenciador no comparte la hipótesis de que se está vulnerando el principio de irrenunciabilidad de los derechos de los trabajadores, -como subterfugio alegado -. De ello, no existe ningún antecedentes objetivo en las pruebas incorporadas por los litigantes.

\subsection{EL PRINCIPIO DE PRIMACÍA DE LA REALIDAD.}

El fallo señaló que, en esta clase de litigios se aprecia la prueba conforme a las normas de la sana crítica, esto es, orientándose el juzgador por los criterios de la lógica y la experiencia, debiendo entenderse integrados a dichas normas los principios formativos del Derecho Laboral, entre los cuales destaca el principio aludido. Por ello se ha dicho que en derecho laboral impera el principio de la primacía de la realidad, según el cual, habrá de estarse a lo que ocurra en la práctica y no a lo que resulte simplemente de los documentos, siempre en función del principio de la buena fe. Así, si bien es cierto que lo obrado por el ente fiscalizador goza de presunción de veracidad, no es menos cierto que esta impida al reclamante desvirtuar aquello con otra prueba -como en la especie ha acontecido-.

3. LA APLICACIÓN DE MÁS DE UNA MULTA POR EL MISMO TIPO DE INFRACCIÓN, LO CUAL SE RELACIONA CON EL PRINCIPIO “NON BIS IN ÍDEM” 
El segundo tema a analizar, se refiere a la vulneración del principio " non bis in ídem”, cuando el fiscalizador aplica más de una multa por la misma infracción, situación que se agrava cuando se trata de grandes empresas ya que, por un lado el artículo 506 del Código del Trabajo establece que si la empresa cuenta con 200 o más trabajadores, cada multa puede llegar a 60 UTM, mientras que en el caso de la micro empresa, el monto máximo será de 10 UTM y por otro lado, habitualmente una gran empresa posee varios establecimientos y si la misma infracción a la normativa laboral, se repite en estos, se pueden aplicar multas por cada uno de ellos, perjudicando gravemente el patrimonio de la empresa.

Consideró que sólo se puede aplicar una multa a la empresa, aunque se constaten las mismas infracciones en las sucursales ya que, el artículo 505 bis, clasifica a las empresas según el número total de trabajadores de ella y no en consideración al número de trabajadores del establecimiento afectado por la multa. Por su parte, el artículo 506, gradúa el monto de las multas según la clasificación referida.

Otra posibilidad de multas que vulneren el principio que se analiza, es que por la infracción de una norma se aplique más de una multa, o un mismo bien jurídico protegido, por ejemplo, se sanciona por no contar con el Reglamento Interno y por no entregarlo a los trabajadores. Estimo que la norma infringida es sólo una, el artículo 184 del Código del Trabajo, que obliga al empleador a tomar medidas eficaces para la protección de la vida y salud de sus trabajadores y a la vez el bien jurídico protegido en las dos multas, es el mismo, la vida y salud de los trabajadores.

He analizado varias sentencias, entre ellas, la correspondiente a la causa RIT: I-22-2015 del Juzgado del Trabajo de Punta Arenas -que es contraria a mi opinión sobre el tema- de la que se desprende que el reclamo se funda en haberse vulnerado el principio non bis in ídem, por cuanto las supuestas infracciones que sustentan las multas se refieren a la protección de la salud de los trabajadores y se ha sancionado por un mismo hecho, es decir por conductas que implican un incumplimiento de las condiciones de seguridad en el trabajo, con varias multas. $\mathrm{Al}$ respecto, señala que deben darse ciertos requisitos para entender que se está vulnerando dicho principio, no bastando que se indique de forma genérica, como lo hace la reclamante, sin detallar como se habría vulnerado en cada una de las infracciones el principio que indica, pues el único argumento de la reclamante es que en ambas fiscalizaciones se cursaron multas por infracciones a las normas de higiene y seguridad, sin mayor detalle, lo que a su juicio es insuficiente para aceptar su argumento de haber sido doblemente sancionado, ya que no explica cómo habría ocurrido esa doble sanción. De aceptarse el argumento de la reclamante, teniendo presente que se trata de una empresa nacional, que presta servicios a JUNAEB en varios establecimientos educacionales a nivel nacional, implicaría que si se le sanciona en uno de ellos, no podrían fiscalizar en ningún otro establecimiento a nivel nacional esa materia, lo que dejaría en indefensión a la mayoría de sus trabajadores y no permitiría que este servicio cumpla con las obligaciones que le impone la ley. 
La sentenciadora señaló que no se dan los requisitos para configurar el denominado principio non bis in ídem, alegado por la empresa, por cuanto las multas fueron cursadas en distintas fiscalizaciones, las que se efectuaron en distintos establecimientos ubicados en distintas ciudades y dicen relación con distintos trabajadores.

\section{EL PLAZO PARA RECLAMAR ADMINISTRATIVAMENTE LAS MULTAS, QUE ESTABLECE EL ARTÍCULO 512 DEL CÓDIGO DEL TRABAJO, ¿ES DE DÍAS CORRIDOS O HÁBILES?}

El último tema de análisis es el referido al plazo para interponer reconsideración administrativa de multas ante la Dirección del Trabajo. Ello, en atención a que el artículo 512 del Código del Trabajo, indica que la resolución que aplica las multas pueden ser reclamadas dentro del plazo de 30 días de notificada la resolución que aplicó la multa administrativa, pero no se señala si el plazo es de días corridos o hábiles, esto es de vital importancia cuando el plazo vence en sábados, domingos o festivos y no se alcanzó a presentar el reclamo dentro de 30 días corridos.

En relación a la materia en análisis, se refieren los fallos Rol N².836-2013, 12.637 2011 y 10.084-2010 de la Excelentísima Corte Suprema y la causa más reciente es la RIT: I30-2015 del Juzgado del Trabajo de Punta Arenas, cuya sentencia acogió el reclamo de la empresa dejando sin efecto las resoluciones que declararon fuera de plazo las reconsideraciones administrativas de multas, en sus fundamentos, la sentenciadora indicó que, el legislador guarda silencio en cuanto a la naturaleza del plazo que consagra y para dilucidarla se acudirá primero a lo que establece el Código del Trabajo en materia de plazos, en el Art. 435 que señala en su inciso final que: "Los términos de días que establece este Título, se entenderán suspendidos los días feriados", sin embargo, como la norma está referida a plazos judiciales previstos para actuaciones en el marco de procedimientos substanciados ante Juzgados de Letras del Trabajo y de Cobranza, no se aplica en la especie, dado que en la controversia que nos ocupa, estamos enfrentados ante un procedimiento administrativo ante la Inspección del Trabajo, en el que se han aplicado multas a la actora y solicitado reconsideración de las mismas. Sin perjuicio de lo dicho, este Tribunal no comparte la posición de la reclamada en orden a que no habiendo norma aplicable al caso en el código del ramo, debe acudirse al derecho común, y aplicarse el Art. 50 del Código Civil, que prescribe en síntesis que los plazos contemplados, entre otros, en las leyes, comprenden los días feriados, salvo que se señale lo contrario, ya que debe tenerse a la vista el principio de la especialidad, para efectos de interpretar la norma. Y lo cierto es que el Código del Trabajo, de por sí es una norma especial, que regula en detalle procedimientos judiciales laborales, pero también contiene cierta normativa referida a instancias administrativas que tienen lugar ante la Dirección del Trabajo, el cual conforme al Art. $1^{\circ}$ del D.F.L. $N^{\circ} 2$ de 1967, es un Servicio técnico dependiente del Ministerio del Trabajo y Previsión Social con el cual se vincula a través 
de la Subsecretaría de Trabajo, en consecuencia, por su naturaleza los procedimientos que ante ella se tramiten, están sujetos, en lo no previsto por la legislación que los regula, a la Ley $\mathrm{N}^{\circ}$ 19.880 que establece Bases de los Procedimientos Administrativos que rigen los actos de los órganos de la Administración del Estado, que se aplica a los servicios públicos creados para el cumplimiento de la función administrativa, todo lo cual fluye de los artículos $1^{\circ}$ y $2^{\circ}$ de la señalada ley. Siendo así las cosas la cuestión controvertida debe resolverse no conforme al derecho común sino aplicando la regla especial del Art. 25 de la ley en comento, que señala: "Cómputo de los plazos del procedimiento administrativo. Los plazos de días establecidos en esta ley son de días hábiles, entendiéndose que son inhábiles los días sábados, los domingos y los festivos. Los plazos se computarán desde el día siguiente a aquél en que se notifique o publique el acto de que se trate o se produzca su estimación o su desestimación en virtud del silencio administrativo. Si en el mes de vencimiento no hubiere equivalente al día del mes en que comienza el cómputo, se entenderá que el plazo expira el último día de aquel mes. Cuando el último día del plazo sea inhábil, éste se entenderá prorrogado al primer día hábil siguiente.”, lo que lleva a concluir que el plazo contemplado en el Art. 512 del Código del Trabajo, para solicitar la reconsideración administrativa de multas impuestas por la Inspección del Trabajo, es de días hábiles, en consecuencia, la presentada el 21 de septiembre del año en curso en contra de las resoluciones de multas de autos, se interpuso dentro del plazo, ya que este vencía recién el día 01 de octubre recién pasado.

\section{CONCLUSIÓN}

En materia de fundamentos de las resoluciones que aplican las multas y su eventual revocación por carecer de ellos se puede establecer que es necesario explicitar la forma en que las conductas del empleador son objeto de un ilícito laboral pues, la sola determinación de un artículo no implica fundamentación en materia de multas. El órgano administrativo fiscalizador del caso no señaló la manera en que entendió cómo las normas laborales se vieron infringidas, pues, como se ha indicado, no es suficiente mencionar un precepto normativo, sino que es necesaria, además, la adecuada fundamentación del mismo.

En el Derecho del Trabajo impera el principio de la primacía de la realidad, según el cual, debe estarse a lo que sucede en los hechos y no a lo que resulte simplemente de los documentos, siempre en función del principio de la buena fe. Así, si bien es cierto que lo realizado por el ente fiscalizador goza de presunción de veracidad, no es menos cierto que esa presunción impida al reclamante desvirtuar aquello con otra prueba -como en la especie ha acontecido.

En cuanto a la aplicación de más de una multa por el mismo tipo de infracción, lo cual se relaciona con el principio "non bis in ídem" es posible concluir que se puede aplicar sólo una multa a las empresas, aunque se constaten las mismas infracciones en las sucursales, 
ya que el artículo 505 bis, distingue a las empresas según el número total de trabajadores de ella y no de acuerdo al número de trabajadores del establecimiento afectado por la multa. Por su parte, el artículo 506, gradúa el monto de las multas según la distinción señalada. Otro peligro de multas que transgredan el principio que se examina, es que por la inobservancia de una norma o un mismo bien jurídico protegido se aplique más de una multa, por ejemplo, se sanciona por no contar con el Reglamento Interno y por no entregarlo a los trabajadores. Aquí el precepto quebrantado es sólo uno, el artículo 184 del Código del Trabajo, que exige al empleador tomar providencias efectivas para el resguardo de la vida y salud de sus trabajadores y a la vez el bien jurídico protegido en las dos multas, es el mismo, la vida y salud de los trabajadores.

En la discusión de si el plazo contemplado en el Art. 512 del Código del Trabajo, para solicitar la reconsideración administrativa de multas impuestas por la Inspección del Trabajo, es de días corridos o hábiles, podemos plantear que constituye un plazo de días hábiles. 